\title{
Recent Advances in our Understanding of Chromospheric and Coronal Heating Mechanisms
}

\author{
Jeffrey L. Linsky ${ }^{1}$ \\ Joint Institute for Laboratory Astrophysics [JILA] \\ University of Colorado \& National Institute of Standards and Technology \\ Boulder, CO 80309-0440, USA
}

\begin{abstract}
I will summarize some of the principal scientific results presented at the Conference on "Mechanisms of Chromospheric and Coronal Heating" held on 5-8 June 1990 in Heidelberg, Germany. The Conference included invited and contributed papers on observations that point to specific heating mechanisms and theoretical papers on the heating mechanisms themselves. There were many opportunities for useful interaction between proponents of these two approaches to understanding stellar chromospheres and coronae. I will concentrate on what is being learned from the empirical side, and then summarize the heating mechanisms discussed and for which types of stars they may be applicable.
\end{abstract}

\section{Introduction}

The organizers of this IAU Colloquium have asked me to summarize some of the principal scientific results presented at the Conference on "Mechanisms of Chromospheric and Coronal Heating" held on 5-8 June 1990 in Heidelberg, Germany. This topic is very germane to the present meeting, since the conversion of mechanical and magnetic energy into heat is the poorly understood intermediate stage that couples the generation of magnetic fields deep inside a star to the observable electromagnetic radiation that we use to study and characterize stellar magnetic activity. While certainly pertinent to the present IAU Colloquium, the mechanisms by which mechanical and magnetic energy may be converted into heat are both numerous and often still poorly understood. I encourage the reader, therefore, to delve deeply into the many excellent review and contributed papers that will appear in the Proceedings of the Heidelberg Conference to be published as Mechanisms of Chromospheric and Coronal Heating. In the meantime, here is my personal summary of some of the highlights of the meeting in a logical progression from the empirical to the theoretical.

\footnotetext{
${ }^{1}$ Staff Member, Quantum Physics Division, National Institute of Standards and Technology
} 


\section{Observations that point to specific heating mechanisms}

\subsection{Chromospheres}

\subsubsection{CO cooling and thermal bifurcation}

Ayres and Testerman (1981) first called attention to the low brightness temperatures observed in the cores of the $\mathrm{CO}$ fundamental $(4.7 \mu \mathrm{m})$ and first overtone $(2.35 \mu \mathrm{m})$ vibration-rotation bands at the solar limb. Subsequent work by Ayres et al. (1986) and a careful non-LTE study of the formation of these molecular lines by Ayres and Wiedemann (1989) left little doubt that the low brightness temperatures in the line cores $\left(T_{b} \leq 3800 \mathrm{~K}\right)$ demonstrate the presence of very cool plasma in the solar chromosphere, a region that was presumed to be a layer with a steeply rising temperature-height distribution. This unexpected result led to a new paradigm of a thermally bifurcated chromosphere consisting of (i) a hot component heated perhaps by magneto-acoustic waves and cooled by $\mathrm{H}^{-}$and ionized metals, and (ii) a cool component having minimal nonradiative heating, cooled by the $\mathrm{CO}$ vibration-rotation bands and presumably a very large number of metallic lines.

Two-component models for the solar atmosphere have now been computed by Ayres, Muchmore, Kneer, and others. Anderson (1989) confirmed that in the absence of nonradiative heating, a radiative equilibrium model for the solar atmosphere will show a monotonic decrease in temperature with height, when a very large number of metallic lines are treated in non-LTE self-consistently. This model is similar to the cool component models computed by Ayres. Also, Anderson and Athay (1989) showed that the addition of a reasonable amount of nonradiative heating to the radiative equilibrium model can reproduce the temperature-height structure of the Maltby et al. (1986) Model C' quite accurately.

Despite this agreement between two-component semiempirical and self-consistent non-LTE model atmospheres, Ayres (1991) mentioned a number of unanswered questions. One is whether the dominant cooling agent in the cool component is $\mathrm{CO}$, as originally thought, or large numbers of iron and other metallic lines. A second question is whether the solar chromosphere is actually thermally unstable.

Ayres pointed out that one sees 200-300 K spatial variations in $\mathrm{T}_{b}$ for the strong CO lines in the $4.7 \mu \mathrm{m}$ band when a 5 " slit is laid across the solar surface. These spatial variations are presumably caused by transient heating events, the $\mathrm{Ca}$ II flashes, that have time scales of roughly $200 \mathrm{~s}$. By comparison, the time scale for cooling by $\mathrm{CO}$ at chromospheric densities, $t_{\text {cool }}(\mathrm{CO}) \sim 1000 \mathrm{~s}$, and the time scale for CO lines to become optically thick, $t_{\tau} \sim 200 \mathrm{~s}$, are independent of the stellar gravity. Since $t_{\text {cool }}(\mathrm{CO})$ is much longer than the transient heating time scale, the response of the solar atmosphere to heating is a rapid rise in temperature, which is observed as the large spatial variations in $\mathrm{T}_{b}$ for the $\mathrm{CO}$ lines. The picture described by Ayres has been criticized by Athay and Dere (1990) who concluded from HRTS data that the chromospheric temperature rise is present over $\sim 90 \%$ of the solar surface.

Muchmore et al. (1987) and others have argued that cooling by $\mathrm{CO}, \mathrm{SiO}$, and perhaps other molecules is thermally unstable in stars across much of the cool half 
of the H-R diagram and that these stars, like the Sun, should show thermally bifurcated chromospheres. Cuntz and Muchmore (1989), for example, computed the propagation of acoustic waves in the atmosphere of Arcturus (KI III). They found that for weak shocks the atmosphere is cool with $\mathrm{CO}$ and $\mathrm{SiO}$ dominating the cooling, whereas strong shocks lead to a hot chromosphere in which $\mathrm{CO}$ and $\mathrm{SiO}$ are not present. Ayres and Wiedemann (1989) have proposed a new two-component semi-empirical model for Arcturus. Johnson (1991) discussed the recent model of the C star TX Pyx computed by Luttermoser et al. (1989) in which the cool component is indicated by $\mathrm{MgI}$ and $\mathrm{CO}$ and the hot chromosphere is indicated by the $\mathrm{Mg}$ II resonance lines in emission. Thus thermal bifurcation appears to be present even in stars with effective temperatures, gravities, and chemical compositions much different from the Sun.

\subsubsection{Chromospheric bright points}

The solar 3-minute chromospheric oscillation seen in time-resolved spectra of the Ca II resonance lines (e.g. Cram and Damé, 1983) has been explained as an upwardpropagating excitation that leads to intense heating in bright points located in the chromospheric network. Rutten (1991) provided a more detailed explanation. $\mathrm{He}$ showed that the intensity fluctuations (seen as $\mathrm{K}_{2 v}$ brightenings) are formed deeper in the chromosphere than the velocity changes (seen as wavelength shifts in the $\mathrm{K}_{3}$ absorption feature). This behavior is consistent with standing waves below the temperature minimum $\left(T_{m i n}\right)$ and running waves above $T_{m i n}$. He suggested that the bright points can be understood as shocks that occur when falling matter (the result of upward propagating waves having insufficient energy to escape the atmosphere) hit the next upward propagating wave. Detailed radiative-hydrodynamic calculations in which a moving piston at the bottom of the atmosphere generates a wave train are needed to understand the data better.

An important question is whether the energy in the cell grains (also called bright points) is sufficient to heat the solar chromosphere. Rutten (1991) said that the answer is yes for the quiet solar chromosphere. Sivaraman (1991) presented new observations in which he found that the bright points all correspond to magnetic network elements (consistent with the earlier work of Sivaraman and Livingston, 1982), but the reverse is not true. He noted that bright points are born where old bright points die, indicating a persistent magnetic geometry. Their lifetimes are 100-200 s and mean magnetic fluxes are 10-20 G. Thus very localized MHD shocks are likely to be major heating mechanisms for the quiet solar chromosphere.

\subsubsection{Chromospheric activity in late-type giants and supergiants}

Pasquini et al. (1991) presented results of their study of 65 stars later than spectral type F8 with luminosity classes ranging from IV to Ib. A comparison of the $\mathrm{Ca}$ II $\mathrm{K}$ line surface fluxes of these stars with stellar masses deduced from evolutionary tracks shows that the more massive stars (initial masses 5-12 $\mathrm{M}_{\odot}$ ) have significantly higher Ca II surface fluxes, and thus higher chromospheric heating rates, than the lower mass giants with $\mathrm{T}_{\text {eff }}<4500 \mathrm{~K}$. 
Their result has important implications for the rate of chromospheric heating in evolved stars. They argue that massive stars start on the main sequence as rapid rotators with no convective zones, but rapid evolution to the right in the $\mathrm{H}-\mathrm{R}$ diagram leads to deep convective zones with minimal loss in angular momentum. Thus dynamo generation of magnetic fields is important for the massive evolved stars and their chromospheres are heated by magnetic processes. By contrast, the lower mass stars had convective zones and lost much of their angular momentum through magnetic winds while on the main sequence and during their slow evolution through the giant branch. Thus their stellar dynamo activity is weak and the chromospheres are heated by nonmagnetic processes at basal flux levels. This picture is not unexpected (e.g. Simon and Drake, 1989), but the Pasquini et al. data set provides the first clear empirical support. For the stars slightly more massive than the Sun, Simon and Drake (1989) find a rapid decline in ultraviolet emission lines near spectral types G0 IV and G0 III which they interpret as due to a transformation from acoustic heating in the early $F$ stars to magnetic heating processes in the cooler stars.

Cuntz and Stencel (1991) called into question this simplistic picture for the least active cool giants like Arcturus. They mentioned the well-known observational result that these stars show variable He I $10830 \AA$ absorption or emission that appears to require either a large amount of plasma at $T_{e} \sim 4 \times 10^{4} \mathrm{~K}$ or photoionization by $x$-rays. There is no evidence as yet in support of either explanation. Recent calculations by Cuntz and Luttermoser (1990) indicate that a stochastic distribution of acoustic wave periods leads to the overtaking and merging of waves. This can occasionally produce very strong shocks with $T_{e} \geq 4 \times 10^{4} \mathrm{~K}$ in the postshock region and absorption in the He I line which vary with time. This may explain the transient He I features within the context of acoustic heating. For these stars the mass loss could be produced by pulsations or acoustic waves with wavelengths similar to a stellar radius (e.g. Cuntz, 1990).

Dupree (1991) noted that episodic heating may explain the formation of the He II $1640 \AA$ line in the Sun and late-type stars. She also suggested that pulsations may be related to the nonmagnetic heating mechanism in the $M$ supergiants like $\alpha$ Ori and in giants near the tip of the red giant branch.

\subsection{Transition regions}

A standard procedure in the analysis of observed line fluxes from solar and stellar transition regions $\left(6.0 \leq \log T_{e} \leq 4.3\right)$ is to determine an emission measuretemperature distribution, $\operatorname{EM}\left(T_{e}\right)=\int_{\Delta T_{e}} n_{e}^{2} d T_{e}$ (e.g. Jordan and Linsky, 1987). This can be done with no assumptions concerning the geometry of the emitting plasma. Jordan (1991) called attention to the very similiar shapes of the $\operatorname{EM}\left(T_{e}\right)$ distributions for dwarf and active giant stars deduced from IUE spectra for emission lines formed within the range $5.3 \leq \log T_{e} \leq 4.3$. She explained this shape similarity as reflecting the radiative properties of the plasma, $\operatorname{EM}\left(T_{e}\right) \sim 1 / P_{\text {rad }}\left(T_{e}\right)$, where $P_{\text {rad }}\left(T_{e}\right)$ is the total power radiated in all spectral features, in the absence of significant local nonradiative heating. Thus the shape of $\operatorname{EM}\left(T_{e}\right)$ provides information on only the redistribution of energy within the transition region and not 
the local heating. Cally (1991) also argued that local heating is not needed in the solar transition region, at least below $10^{5} \mathrm{~K}$, because the observed $\operatorname{EM}\left(T_{e}\right)$ distribution can be explained by the balance of turbulent conductivity heat transport with radiative losses.

Feldman (1983), Habbal (1991) and others have used Skylab far-ultraviolet spectra to argue that most of the solar emission measure in the temperature range $5.3 \leq \log T_{e} \leq 4.5$ originates in cool loops that are magnetically isolated from the chromosphere and corona, although a small portion of the emission originates in a "classical" transition region at the base of hot magnetic loops. Habbal (1991) also noted that the greatest fractional variability occurs in the O IV line formed at 1-2 $\times 10^{5} \mathrm{~K}$, primarily in bright points located over the chromospheric network. Thus the inference of heating rates and details of the heating mechanism(s) for the solar transition region are complicated by the complex geometry.

\subsection{Coronae}

\subsubsection{Are stellar coronae heated by acoustic waves?}

Until the Einstein observations of late-type stars became available, the most popular theories for the heating of solar and stellar coronae assumed the dissipation of purely acoustic or magneto-acoustic waves. In his review talk, Rosner (1991) summarized the main arguments against the purely acoustic wave heating theory. One is that the deduced x-ray surface fluxes for late-type stars correlate well with stellar rotation period and rotational velocity but poorly with $T_{\text {eff }}$ and gravity, the two parameters that determine the properties of the convective zone where acoustic waves are generated. A second argument is that the $\mathrm{x}$-ray emission for the Sun is brightest where the magnetic field is strongest, and the highest spatial resolution solar images in coronal emission lines reveal bright coronal loops that presumably trace closed magnetic field lines (Golub, 1991). Jordan (1991) provided a third argument - measured nonthermal line widths for lines formed in the chromosphere and transition region indicate a mean wave energy of $5 \times 10^{5} \mathrm{erg}$ $\mathrm{cm}^{-2} \mathrm{~s}^{-1}$, which is insufficient to heat the lower corona of the Sun either by acoustic or by magnetic wave modes. Calculations by Stẹpień and Ulmschneider (1989) and by Hammer and Ulmschneider (1990) confirm that pure acoustic waves can heat the atmospheres of late-type stars to coronal temperatures, but these coronae would have very low base pressures and be unobservable.

\subsubsection{The coronal dividing line in the $H-R$ diagram}

Using the early x-ray observations from the Einstein satellite, Ayres et al. (1981) first called attention to a rather sharp dividing line in the $\mathrm{H}-\mathrm{R}$ diagram separating the $G$ giants, which were typically detected as $x$-ray sources and thus have hot coronae, from the later $\mathrm{K}$ and $\mathrm{M}$ giants which were not detected as $\mathrm{x}$-ray sources and thus have little or no plasma at coronal temperatures. In fact, the upper limit on the x-ray surface flux from the nearby K1 III star Arcturus is 1000 times smaller than is observed from the quiet Sun (Ayres et al., 1982). Zwaan (1991) noted that 
this picture has not changed in the past 9 years (cf. Maggio et al., 1990; Haisch et al., 1990), but ROSAT observations of the noncoronal stars are awaited to test it. Zwaan's (1991) explanation for the absence of hot plasma in the cooler giants is the same evolutionary argument proposed by Pasquini et al. (1991) to explain the weak chromospheric emission from these stars. Thus magnetic heating processes should be very weak for the cool giants, and acoustic waves can heat only an unobservably small amount of material to coronal temperatures.

\subsubsection{Overactive close binaries}

The dependence of many activity indicators, such as the x-ray surface flux, on rotational period or Rossby number has been confirmed by many authors and is one of the major arguments for the magnetic nature of the heating processes in rapidly rotating stars. In his review talk, Zwaan (1991) called attention to an anomaly in this behavior; namely, the $\mathrm{G}$ and $\mathrm{K}$ subgiant components in close binary systems are "overactive" in the sense that they have x-ray surface fluxes much larger than single stars with the same rotational periods. The close binaries are rapid rotators because tidal interactions produce synchronism between the orbital and rotational periods. His explanation for "overactivity" is that the tidal forces also produce a different differential rotation structure than is present naturally in a single star, and this altered angular momentum distribution leads to enhanced dynamo generation of magnetic fields. Rodonò (1986), for example, summarizes data on RS CVn systems that shows that the differential rotation with latitude in these stars is typically 1-3 orders of magnitude smaller than for the Sun.

\subsubsection{Why are the coronae of active stars so hot?}

Einstein Solid State Spectrometer observations of RS CVn systems (Swank et al., 1981) and the dMe star Wolf $630 \mathrm{AB}$ (Swank and Johnson, 1982) indicate that a substantial portion or in some cases most of the coronal emission measure at times outside of identifiable flares is at very high temperatures $\left(7.4 \leq \log T_{e} \leq\right.$ 7.8). During flares, the observed x-ray emission indicates even hotter plasma; for example, EXOSAT observations of the 29 September 1983 flare on the RS CVn system $\sigma^{2} \mathrm{CrB}$ (van den Oord et al., 1988) indicate $T_{e}=9.5 \times 10^{7} \mathrm{~K}$. Rosner (1991) asked why these active stars have coronal plasma temperatures more than an order of magnitude hotter than is observed in solar active region loop and even hotter than is observed during the thermal phases of most solar flares. He did not answer his question, but he did note that the solar x-ray luminosity is near the bottom of the observed stellar $\mathrm{L}_{x}$ distribution.

In my view it is important to know that those stars with very hot plasma temperatures generally are observed to be luminous microwave sources, produced by gyrosynchrotron emission from a time varying distribution of relativistic electrons. Thus the coronal heating process is probably closely related to the process that accelerates electrons to relativistic energies. The most likely candidate is some type of magnetic reconnection process that creates hot thermal electrons at the same time as the relativistic electrons or as a thermalization byproduct. 


\subsubsection{Solar coronal oscillations}

Another clue concerning coronal heating processes comes from Pasachoff's (1991) observations of enhanced power in the $5303 \AA$ line near $1 \mathrm{~Hz}$ during the 1980 solar eclipse he observed in India and at $0.2-3 \mathrm{~Hz}$ during the June 1983 eclipse observed in Indonesia. He suggested that the observed oscillatory power refers to the reflection of MHD waves at the mirroring points in coronal magnetic loops. The observed timescales are consistent with either transverse or torsional body waves in these loops, as discussed by Hollweg (1991) and Berger (1991), or the LRC circuit model discussed by Cram (1991) at the meeting.

\subsection{Flux-flux relations and basal and saturated heating}

A critical step in determining the heating processes at work in stellar chromospheres and coronae is an accurate measurement of the total radiative loss from the plasma within a specified temperature range. This is difficult since many spectral lines and continua contribute to the total radiative loss. Linsky (1991) reviewed some of the spectral diagnostic problems encountered in deriving differential emission measures and methods for including all major emitters, especially Fe II in the chromosphere, in determining the total power radiated from a plasma. $\mathrm{He}$ proposed two scaling laws by which one may simply determine the total power radiated by the chromosphere (from the $\mathrm{Mg} \mathrm{II} \mathrm{h}$ and $\mathrm{k}$ lines) and by the higher temperature layers (from the C IV $1550 \AA$ feature).

\subsubsection{Basal fluxes}

The heating of stellar chromospheres appears to consist of two components - an active component for which the heating rate depends on stellar rotation, and a basal component that depends on $\mathrm{T}_{e f f}$ and perhaps only weakly on gravity. In his review of this topic, Schrijver (1987a) showed that the basal radiative loss rates can be measured as lower limits to the surface fluxes in the $\mathrm{Ca}$ II and $\mathrm{Mg}$ II resonance lines and the Si II $1812 \AA$ multiplet in flux-color diagrams as functions of stellar B-V color. Zwaan (1991) extended this work by showing that the sum of the minimum radiative fluxes in these lines can be used to infer the total radiative loss rate from a basal chromosphere and thus the basal heating rate, $F_{b a s a l} \sim T_{e f f}^{\gamma}$.

This empirical heating rate may be compared with the theoretical rates for heating by pure acoustic waves computed by Bohn (1984), although Musielak (1991) mentioned that stellar acoustic fluxes are still poorly known. One should understand that (magneto-)acoustic energy fluxes are computed for the top of a stellar convective zone and thus cannot be simply compared with observed chromospheric energy losses, since most of the wave energy is dissipated by radiation in the photosphere. Thus acoustic waves are the most viable heating mechanism for the chromospheres of the least active stars, including the early $\mathrm{F}$ dwarfs, slowly rotating $\mathrm{G}-\mathrm{M}$ dwarfs, cool giants which are not Miras, $M$ supergiants, and the centers of solar supergranulation cells, which have chromospheric line surface fluxes close to the basal values. Judge and Stencel (1991) recently concluded that the 
Table 1. A summary of proposed heating mechanisms and where they may be important

\begin{tabular}{|c|c|}
\hline Proposed Heating Mechanism & Where It May Be Important \\
\hline $\begin{array}{l}\text { (1) Short period acoustic waves } \\
\text { - Stochastic heating (Cuntz) } \\
\text { - Basal heating (Ulmschneider) } \\
\text { - Multipole sources (Musielak) } \\
\end{array}$ & $\begin{array}{l}\text { - Weak basal heating for late-type stars. } \\
\text { Dominant heating for cool giants, M supergiants. } \\
-P \sim P_{A} / 10 \text { to } P_{A} / 5 \Rightarrow \text { shock heating } \\
\text { in chromospheres but not coronae. }\end{array}$ \\
\hline $\begin{array}{l}\text { (2) 3- to 5-minute oscillations } \\
\text { with large phase shifts } \\
\text { (pure acoustic modes) }\end{array}$ & $\begin{array}{l}\text { - Standing waves in solar photosphere } \\
\text { do not } \Rightarrow \text { shocks in solar chromosphere. } \\
\text { - Heating in cool giants (i.e. Miras) may } \Rightarrow \text { mass loss. }\end{array}$ \\
\hline $\begin{array}{l}\text { (3) Strong acoustic shocks } \\
\text { strengthened by radiative amplification } \\
\text { (radiative instabilities) }\end{array}$ & $\begin{array}{l}\text { - May heat density inhomogeneities } \\
\text { in OB star winds. }\end{array}$ \\
\hline $\begin{array}{l}\text { (4) Resistive current dissipation } \\
\text { following magnetic field reconnection }\end{array}$ & $\begin{array}{l}\text { - Coronae of rapidly rotating late-type stars. } \\
\text { - Heating rate depends on } v_{\text {rot. }}\end{array}$ \\
\hline $\begin{array}{l}\text { (5) MHD wave dissipation processes } \\
\text { in general } \\
\text { (Musielak) }\end{array}$ & $\begin{array}{l}\text { - Coronae of rapidly rotating cool stars. } \\
\text { - Heating rate depends on } v_{\text {rot }} \text {. } \\
\text { - Generated by turbulent and convective motions } \\
\text { in convection zones. } \\
\text { - Energy transmitted by motions of footpoints } \\
\Rightarrow \text { heating of coronal magnetic structures. } \\
\text { - Energy input also from magnetic fluxtube emergence. }\end{array}$ \\
\hline $\begin{array}{l}\text { (5a) MḦD fast mode waves } \\
\text { (Stein) }\end{array}$ & $\begin{array}{l}\text { - May heat coronal loops when waves produced locally. } \\
\text { - Generated by mode-mode coupling. }\end{array}$ \\
\hline $\begin{array}{l}\text { (5b) Transverse and torsional } \\
\text { Alfvén body waves } \\
\text { (Hollweg, Berger) }\end{array}$ & $\begin{array}{l}\text { - Propagate readily through the corona. } \\
\text { - Can heat coronal loops by resonances, phase mixing, } \\
\text { mode coupling, turbulence, and Landau damping. }\end{array}$ \\
\hline $\begin{array}{l}\text { (5c) Slow mode MHD waves and } \\
\text { longitudinal tube waves } \\
\text { (magneto-acoustic type waves) } \\
\text { (Stein) }\end{array}$ & $\begin{array}{l}\text { - Propagate from convective zone to chromosphere. } \\
\text { - Radiative damping in photosphere and chromosphere. } \\
\text { - Heat the low and middle chromosphere } \\
\text { by dissipation of shocks. } \\
\text { - Easily generated by mode coupling from other waves. }\end{array}$ \\
\hline $\begin{array}{l}\text { (5d) Alfvénic surface waves } \\
\text { (Roberts) }\end{array}$ & $\begin{array}{l}\text { Heat coronal loops by resonant absorption } \\
\text { in thin sheaths. }\end{array}$ \\
\hline (5e) Shear Alfvén waves & $\begin{array}{l}\text { - Generated by phase mixing, mode-mode coupling, } \\
\text { and turbulent heating. } \\
\text { - May heat coronal holes. } \\
\text { - Heating } \Rightarrow \text { thermal energy to drive solar wind. } \\
\text { - Adds momentum to high speed solar streamers. }\end{array}$ \\
\hline $\begin{array}{l}\text { (6) Anomalous current dissipation and } \\
\text { and magnetic reconnection } \\
\text { (Priest, Spicer, Jardine) }\end{array}$ & $\begin{array}{l}\text { - May heat coronal loops. In vortex structures? } \\
\text { - Intermittent heating mechanisms. } \\
\text { - Heating in thin sheets or layers. }\end{array}$ \\
\hline $\begin{array}{l}\text { (7) Resonant LRC circuit } \\
\text { (Cram) }\end{array}$ & - Useful formalism. \\
\hline $\begin{array}{l}\text { (8) Microflares and nanoflares } \\
\text { (Parker) }\end{array}$ & $\begin{array}{l}\text { - Same as current dissipation. } \\
\text { - May heat coronal loops quasi-statically. }\end{array}$ \\
\hline (9) Large scale flows & $\begin{array}{l}\text { - Flows in spicules. } \\
\text { - Initiated by other heating mechanisms. }\end{array}$ \\
\hline
\end{tabular}

basal flux limits deduced by Schrijver (1987b) can be extrapolated to giant stars with very low effective temperatures. 


\subsubsection{Saturated fluxes}

At the opposite extreme, there appears to be maximum values for the observed surface fluxes in the chromospheric resonance lines of $\mathrm{Ca}$ II and $\mathrm{Mg}$ II, transition region lines like C IV $1550 \AA$ and the coronal soft x-ray flux. Vilhu (1987) and Vilhu and Walter (1987) showed that these saturated fluxes occur in the most rapidly rotating stars of a given color. The saturated surface fluxes could be interpreted as those corresponding to the maximum rate of steady-state heating possible given the available mechanical energy in the convective motions and complete surface coverage by equipartition magnetic fields (about $1500 \mathrm{G}$ in the solar photosphere). Schrijver (1987a,b) noted that for solar plages the $\mathrm{Mg}$ II surface fluxes are a factor of 10-30 below the saturated values, while typical magnetic flux densities are about a factor of 15 below the equipartition values, implying that strong magnetic fields only fill about $10 \%$ of the surface area. On the other hand, the heating rates for plages in RS CVn systems are consistent with saturated heating (Linsky 1991) both in the chromosphere and in the higher temperature layers.

Schrijver (1991) called attention to empirical scaling laws of the form, $F_{\text {diagnostic }}=|B|^{\alpha}$, which relate the solar and stellar surface fluxes in a specific spectral diagnostic to the magnetic flux density either for small regions on the solar surface or a mean value for stars with $|B|=f B$, where $f$ is the magnetic filling factor and $B$ the magnetic field strength determined using Zeeman broadening techniques (e.g. Saar, 1987). The value of $\alpha$ increases from 0.6 for the Ca II K line and $\mathrm{Mg} \mathrm{II}$, to 0.7 for $\mathrm{C} \mathrm{IV}$, and to 1.0 for coronal $\mathrm{x}$-ray emission. Thus the chromospheric diagnostics do not count magnetic flux tubes. Schrijver (1991) argues that the change in $\alpha$ with temperature indicates that the heating mechanisms for the solar chromosphere and corona are probably different.

\subsubsection{Explanations for flux-flux relations}

Using the early observations from IUE and Einstein, Ayres, Marstad and Linsky (1981) showed that the normalized fluxes of diagnostics formed at different temperatures can be fit by power laws in which the index becomes larger as the temperature difference at which the diagnostics are formed increases. Zwaan (1991) reviewed more recent work that shows that tighter power law relations follow when basal fluxes are subtracted from the surface fluxes of chromospheric lines, i.e. $\mathrm{F}_{\text {diagnostic }} \sim\left(\mathrm{F}_{\text {chrom }}-\mathrm{F}_{\text {basal }}\right)^{\beta}$. The quantity $\beta\left(T_{e}\right)$, which increases with temperature, can be viewed as an activity parameter. Zwaan did not propose an explanation for the temperature dependence of $\beta\left(T_{e}\right)$, but Jordan (1991) argued that local heating is unimportant in the lower transition region $\left(\mathrm{T}_{e} \leq 2 \times 10^{5}\right.$ $\mathrm{K})$. Therefore, the shape of $\mathrm{EM}\left(\mathrm{T}_{e}\right) \sim P_{\text {rad }}^{-1}\left(T_{e}\right)$ and the flux-flux plots provide information only on how energy is redistributed in the lower transition region and not on the local heating mechanism. 


\section{Chromospheric and coronal heating mechanisms}

The remainder of the meeting was devoted to presentations of the many different mechanisms that may heat stellar chromospheres and coronae. I do not have the space to summarize these presentations in detail, but I include in Table 1 a concise summary of the different mechanisms discussed by the various speakers (their names are in bold type) and in what types of stars they may be important. A more complete description of these mechanisms may be found in the recent review paper by Narain and Ulmschneider (1990).

Acknowledgements. This work is supported by NASA grants NAG6-82 to the University of Colorado and S-56460-D to the National Institute of Standards and Technology. I would like to thank Dr. M. Cuntz for his comments on the manuscript.

\section{References}

Anderson, L.S.: 1989, Astrophys. J. 339, 558.

Anderson, L.S., Athay, R.G.: 1989, Astrophys. J. 346, 1010.

Athay, R.G., Dere, K.P.: 1990, Astrophys. J. 358, 710.

Ayres, T.R.: 1991, see Ulmschneider et al.

Ayres, T.R., Simon, T., Linsky, J.L.: 1982, Astrophys. J. 263, 791.

Ayres, T.R., Linsky, J.L., Vaiana, G.S., Golub, L., Rosner, R.: 1981, Astrophys. J. 250, 293.

Ayres, T.R., Marstad, N.C., Linsky, J.L.: 1981, Astrophys. J. 247, 545.

Ayres, T.R., Testerman, L.: 1881, Astrophys. J. 245, 1124.

Ayres, T.R., Testerman, L., Brault, J.W.: 1986, Astrophys. J. 304, 542.

Ayres, T.R., Wiedemann, G.: 1989, Astrophys. J. 338, 1033.

Berger, M.A.: 1991, see Ulmschneider et al.

Bohn, U.H.: 1984, Astron. Astrophys. 136, 338.

Cally, P.S.: 1991, see Ulmschneider et al.

Cram, L.E.: 1991, see Ulmschneider et al.

Cram, L.E., Damé, L.: 1983, Astrophys. J. 272, 355.

Cuntz, M.: 1990, Astrophys. J. 353, 255.

Cuntz, M., Luttermoser, M.: 1990, Astrophys. J. Letters 353, L39.

Cuntz, M., Muchmore, D.: 1989, Astron. Astrophys. 209, 305.

Cuntz, M., Stencel, R.E.: 1991, see Ulmschneider et al.

Dupree, A.K.: 1991, see Ulmschneider et al.

Feldman, U.: 1983, Astrophys. J. 275, 367.

Golub, L.: 1991, see Ulmschneider et al.

Habbal, S.R.: 1991, see Ulmschneider et al.

Haisch, B.M., Bookbinder, J.A., Maggio, A., Vaiana, G.S., Bennett, J.O.: 1990, Astrophys. $J$., in press.

Hammer, R., Ulmschneider, P.: 1990, in Cool Stars, Stellar Systems, and the Sun, ed. G. Wallerstein (San Francisco: Astronomical Soc. Pacific), p. 51.

Hollweg, J.: 1991, see Ulmschneider et al.

Johnson, H.R.: 1991, see Ulmschneider et al. 
Jordan, C.: 1991, see Ulmschneider et al.

Jordan, C., Linsky, J.L.: 1987, in Exploring the Universe with the IUE Satellite, ed. Y.

Kondo et al. (Dordrecht: Reidel), p. 259.

Judge, P., Stencel, R.E.: 1991, Astrophys. J., in press.

Linsky, J.L.: 1991, see Ulmschneider et al.

Luttermoser, D.G., Johnson, H.R., Avrett, E.H., Loeser, R.: 1989, Astrophys. J. 345, 543.

Maggio, A., Vaiana, G.S., Haisch, B.M., Stern, R.A., Bookbinder, J., Harnden, F.R. Jr., Rosner, R.: 1990, Astrophys. J. 348, 253.

Maltby, P., Avrett, E.H., Carlsson, M., Kjeldseth-Moe, O., Kurucz, R.L., Loeser, R.: 1986, Astrophys. J. 306, 284.

Muchmore, D., Nuth III, J.A., Stencel, R.E.: 1987, Astrophys. J. Letters 315, L141.

Musielak, Z.: 1991, see Ulmschneider et al.

Narain, U., Ulmschneider, P.: 1990, Space Science Reviews, in press.

Pasquini, L., Brocato, E., Pallavicini, R.: 1991, see Ulmschneider et al.

Pasachoff, J.M.: 1991, see Ulmschneider et al.

Rosner, R.: 1991, see Ulmschneider et al.

Rodonò, M.: 1986, in Cool Stars, Stellar Systems, and the Sun, ed. M. Zeilik and D.M. Gibson, Springer-Verlag, Berlin, p. 475.

Rutten, R.J. 1991, see Ulmschneider et al.

Saar, S.H.: 1987, in Cool Stars, Stellar Systems, and the Sun, Lecture Notes in Physics 291, ed. J.L. Linsky, R.E. Stencel, Springer-Verlag, Berlin, p. 10.

Schrijver, C.J.: 1987a, in Cool Stars, Stellar Systems, and the Sun, Lecture Notes in Physics 291, ed. J.L. Linsky, R.E. Stencel, Springer-Verlag, Berlin, p. 135.

Schrijver, C.J.: 1987b, Astron. Astrophys. 180, 241.

Schrijver, C.J.: 1991, see Ulmschneider et al.

Simon, T., Drake, S.A.: 1989, Astrophys. J. 346, 303.

Sivaraman, K.R.: 1991, see Ulmschneider et al.

Sivaraman, K.R., Livingston, W.C.: 1982, Solar Phys. 82, 227.

Stępień, K., Ulmschneider, P.: 1989, Astron. Astrophys. 216, 139.

Swank, J.H., Johnson, H.M.: 1982, Astrophys. J. Letters 259, L67.

Swank, J.H., White, N.E., Holt, S.S., Becker, R.H.: 1981, Astrophys. J. 246, 208.

van den Oord, G.H.J., Mewe, R., Brinkman, A.C.: 1988, Astron. Astrophys. 205, 181.

Ulmschneider, P., Priest, E., Rosner, R. (eds.): Mechanisms of Chromospheric and Coronal Heating, Springer-Verlag, Berlin, in press.

Vilhu, O.: 1987, in Cool Stars, Stellar Systems, and the Sun, Lecture Notes in Physics 291, ed. J.L. Linsky, R.E. Stencel, Springer-Verlag, Berlin, p. 110.

Vilhu, O., Walter, F.M.: 1987, Astrophys. J. 321, 958.

Zwaan, C.: 1991, see Ulmschneider et al. 